\title{
Frecuencia cardiaca y actividad física en niños con obesidad del Sur de Sonora
}

\section{Heart rate and physical activity among obese children in south Sonora}

\section{Frequência cardíaca e atividade física em crianças com obesidade do Sul de Sonora}

\author{
J.Valle-Leal ${ }^{\mathrm{a}, *}$, R. García-Moreno ${ }^{\mathrm{b}}$, M. Espinoza -Salazar ${ }^{\mathrm{b}}$, \\ C.O. Muñoz-Robles ${ }^{\text {b }}$, B. Dennis-Yepiz ${ }^{\text {, }}$, D.B. Orduño-Felician ${ }^{c}$ \\ ${ }^{a}$ Departamento de Pediatría, Hospital General Regional Número Uno del Instituto Mexicano del Seguro Social \\ (IMSS), Ciudad Obregón, Sonora, México \\ ${ }^{\text {b }}$ Departamento de Enfermería, Hospital General Regional Número Uno del Instituto Mexicano del Seguro So- \\ cial (IMSS), Ciudad Obregón, Sonora México \\ ${ }^{\mathrm{c}}$ Escuela de Enfermería, Instituto Mexicano del Seguro Social, Ciudad Obregón, Sonora, México
}

Recibido: 9 enero 2018

Aceptado: 13 agosto 2018

\section{Resumen}

Introducción: La obesidad infantil provoca una alteración en la modulación autonómica cardiaca. Se ha señalado que la reducción de la acción protectora del sistema nervioso parasimpático cardiaco, manifestada en una frecuencia cardiaca más acelerada tras la actividad física en este grupo de pacientes, podría representar un dato temprano de disfunción cardiovascular.

Objetivo: Determinar diferencias en la Frecuencia cardiaca en reposo y tras actividad física en escolares con y sin obesidad, adscritos al servicio de pediatría de un Hospital de Segundo Nivel de Atención.

Material y método: Bajo un diseño transversal, y un muestreo no probabilístico; se estudiaron niños en edad escolar que asistían a consulta externa de pediatría, se categorizó estado nutricional mediante score Z de IMC e índice cintura-estatura, se midió frecuencia cardiaca en tres momentos: reposo, posterior a actividad y en reposo tras actividad. Se realizó análisis descriptivo de las variables, coeficiente de correlación de Pearson, Kruskal Wallis y U de Mann Whitney, para medir asociación y diferencia de frecuencias cardiacas entre los grupos estudiados.

Resultados: Se estudiaron 106 niños en edad escolar, el 53\% presentaba obesidad abdominal y el 66\% sobrepeso/obesidad. Se encontraron diferencias de medias estadísticamente significativas en las frecuencias cardiacas post actividad y tras reposo post actividad, entre pacientes con y sin obesidad. 
Discusión y Conclusiones: Los niños con sobrepeso y obesidad deberían tener un seguimiento estrecho, ya que el marcado aumento de la FC después de la actividad física, debe ser tomado en cuenta como signo de alarma temprano de disfunción cardiovascular.

Palabras clave: Obesidad infantil; frecuencia cardiaca; obesidad abdominal; actividad física; México.

\section{Abstract}

Introduction: Childhood obesity provokes alterations in the cardiac autonomic modulation; and it has been pointed that disturbances to cardiac parasympathetic system, manifested as faster heart rate after physical activity, in this group of patients, could represent an early sign of cardiovascular disfunction.

Objective: To determine differences in heart rate at rest and after physical activity among school children, with and without obesity, attending to a pediatric service in a Second Level of Attention Hospital.

Materials and method: Under a transversal study design, and non-probabilistic sampling, school children attending to pediatric service were studied. The nutritional status was assessed through BMI Z scores, and a waist/height index. Heart rate were measured at three times: rest, after some physical activity, and at rest after such physical activity. A descriptive analysis was performed, and Pearson correlation coefficient, and Kruskal-Wallis, and Mann-Whitney tests were calculated to assess cardiac-related associations and differences between the two groups.

Results: 106 school children were studied. 53\% showed abdominal obesity and 66\% overweight/obesity. Statistically significant mean differences were found in the cardiac frequencies post activity and at rest post activity between the patients with and without obesity.

Discussion and conclusion: Considering the notorious increases in cardiac frequencies after physical activity, children with overweight and obesity should to be closely monitored for possible signs of cardiovascular disfunction.

Keywords: Child obesity; heart rate; abdominal obesity; physical activity; Mexico.

\section{Resumo}

Introdução: A obesidade infantil provoca uma alteração na modulação autonómica cardíaca. Foi apontado que a redução da ação protetora do sistema nervoso parassimpático cardíaco, manifestada em uma frequência cardíaca mais acelerada após a atividade física neste grupo de pacientes, poderia representar um dado precoce de disfunção cardiovascular neles.

Objetivo: Determinar diferenças na Frequência cardíaca em repouso e após da atividade física em estudantes com e sem obesidade, vinculados ao serviço de pediatria de um Hospital de Segundo Nível de Atenção.

Material e método: Sob um desenho transversal, e uma amostra não probabilística; estudaram-se crianças em idade escolar que assistiam a consulta externa de pediatria, categorizou-se estado nutricional mediante score Z de IMC e índice cinto-estatura, mediu-se frequência cardíaca em três momentos: repouso, posterior à atividade e em repouso após a atividade. Realizou-se análise descritiva das variáveis, coeficiente de correlação de Pearson, Kruskal Wallis e U de Mann Whitney, para medir associação e diferença de frequências cardíacas entre os grupos estudados.

Resultados: Estudaram-se 106 crianças em idade escolar, o 53\% apresentava obesidade abdominal e o 66\% sobrepeso/obesidade. Encontraram-se diferenças de médias estatisticamente significativas nas frequências cardíacas post atividade e após repouso post atividade, entre os pacientes com e sem obesidade.

Discussão e Conclusões: As crianças com sobrepeso e obesidade deveriam ter um acompanhamento maior, já que o marcado aumento da FC depois da atividade física é um signo de alarme, que deve ser tomado em conta como signo precoce de disfunção cardiovascular.

Palavras chave: Obesidade infantil; frequência cardíaca; obesidade abdominal; atividade física; México. 


\section{Introducción}

El paciente pediátrico debe valorarse integralmente por parte de personal médico y de enfermería en cada oportunidad que se tenga, esto incluye escenarios como consultorios de unidades médicas, guarderías y servicios de medicina preventiva. Parte importante dentro de la atención que debe otorgarse a estos pacientes son las mediciones somatométricas que incluyen peso, estatura, perímetro de cintura y la medición de constantes vitales como presión arterial (PA) y frecuencia cardiaca (FC). La medición y clasificación de peso, así como el perímetro de cintura deben realizarse en todo niño para clasificar su estado nutricional de acuerdo al Índice de Masa Corporal (IMC) y el índice de cintura/estatura. La medición de constantes vitales refleja el estado fisiológico de órganos como: corazón, cerebro, pulmones ${ }^{1}$, esto es una actividad clave en la valoración, diagnóstico e implementación de medidas terapéuticas por parte de los profesionales de la salud. La medición de la FC y PA requiere precisión en la técnica, debe ajustarse a la edad y estatura del niño, la estandarización de estas constantes vitales es importante en la detección de múltiples patologías dentro las que destacan las cardiovasculares².

La obesidad infantil se caracteriza por un exceso de adiposidad determinado por un Índice de Masa Corporal (IMC), igual o mayor al percentil 95 para edad y género ${ }^{3,4}$. En México según resultados de la encuesta de salud y nutrición (ENSANUT) 2012, la prevalencia combinada de obesidad y sobrepeso para niños menores de 5 años fue del $9.7 \%$, en niños entre 5 y 11 años $34.4 \%$ y para adolescentes el dato fue de $34.9 \%^{5}$. ENSANUT 2016 reportó un 33.2\% prevalencia combinada de sobrepeso y obesidad en niños de 6 a 11 años, $36.3 \%$ en adolescentes, en esta última encuesta se incrementó el número de casos en poblaciones rurales ${ }^{6}$.

La obesidad se asocia a múltiples patologías metabólicas y cardiacas entre las que destacan la diabetes mellitus e hipertensión arterial. Se han encontrado en pacientes pediátricos con obesidad, alteraciones del sistema nervioso autónomo (SNA), el cual es un importante controlador involuntario de muchos procesos fisiológicos, entre ellos: la FC, contractilidad miocárdica y la PA; esta regulación la ejerce a través de los sistemas simpático y parasimpático ${ }^{5}$. En algunos estudios se ha reportado un predominio del sistema simpático sobre el parasimpático en niños con obesidad ${ }^{7,8}$.

La FC es uno de los parámetros no invasivos más utilizados en el análisis y valoración de la modulación cardiaca autonómica, en niños con obesidad se ha encontrado una limitación en la recuperación de ésta tras el ejercicio físico, así como un menor rendimiento aeróbico ${ }^{9-12}$.

Cuando el organismo se expone a cambios fisiológicos como el ejercicio físico, la regulación cardiovascular está basada en un balance de las dos ramas del sistema nervioso autónomo, en este caso un balance dinámico del sistema nervioso simpático y parasimpático, proceso llamado balance Simpático-Vagal ${ }^{12}$.

La reducción en la actividad parasimpática ha sido identificada como una causa importante de morbilidad y mortalidad cardiovascular, por lo que la identificación temprana de la desregulación del sistema nervioso autónomo en niños con obesidad, podría ser un marcador clínico importante para la prevención de aparición de enfermedades cardiovasculares en este grupo de pacientes ${ }^{13}$.

El ejercicio físico es parte de los pilares del tratamiento del niño con obesidad y sobrepeso, pero es importante considerar las implicaciones que el exceso de adiposidad pueda tener sobre su respuesta cardiaca, ya que puede modificar de manera importante la respuesta de este órgano ante la actividad física, es pues una necesidad en todo paciente, antes de someterlo a un aumento de actividad física ser valorado integralmente por parte de personal médico y de enfermería, con la finalidad de anticiparse a conocer la respuesta cardiovascular y así poder determinar la intensidad del ejercicio a que se someterá el paciente ${ }^{14-16}$.

Existen pocos estudios nacionales, donde se haya medido la respuesta cardiaca tras la actividad física en niños asociada al estado nutricional, por lo que el objetivo de este estudio fue determinar la frecuencia cardiaca en reposo y tras actividad física en escolares con y sin obesidad, adscritos al servicio de pediatría de un Hospital de Segundo Nivel de Atención del Sur de Sonora.

Frecuencia cardiaca y actividad física en niños con obesidad del Sur de Sonora • J. Valle-Leal et al. 


\section{Material y método}

Previa autorización por el comité de ética e investigación, consentimiento informado de los padres y asentimiento por parte del paciente, el estudio se realizó bajo un diseño transversal en pacientes pediátricos de edad escolar (6-12 años).

El cálculo del tamaño de la muestra se realizó para un poder estadístico del 95\%. El muestreo se llevó acabo por casos consecutivos; se incluyó a pacientes de 6-12 años de edad, género indistinto, que acudían a la consulta de pediatría de un Hospital de Seguridad Social, de segundo nivel de atención en el noroeste de México, la muestra quedó constituida por 106 niños. Se excluyeron aquellos pacientes con cardiopatías, endocrinopatías, genopatías, neumopatías o que presentaban alguna limitación física, que impidiera realizar ejercicio físico de intensidad moderada, y también aquellos pacientes con práctica de actividad física de moderada a fuerte intensidad de manera regular, durante los últimos 6 meses. Los sujetos estudiados pertenecen en su mayoría a clase social media y baja, acuden a escuelas públicas ubicadas en área urbana en las cuales no existe personal médico o de enfermería y en donde no se llevan a cabo actividades para la prevención de enfermedades, en estas instituciones la práctica de actividad física reglamentaria es de 1 a 2 horas por semana.

A cada participante se le realizaron los siguientes procedimientos, entrevista para obtención de edad y género, somatometría que incluía peso y talla para cálculo de IMC mediante formula de Quetelet, perímetro de cintura para cálculo de índice de cintura-estatura, estas mediciones se realizaron según lineamientos emitidos en el manual de antropometría del departamento de Nutrición del Instituto de Ciencias Médicas y Nutrición Salvador Zubirán ${ }^{13}$. Se calculó score Z de IMC según edad y género, se clasificó el estado nutricional según los criterios de OMS, $<1$ desviación estándar (DS) = peso normal, sobrepeso igual o $>$ de 1 a 2 DS y obesidad cuando el resultado fuera $>$ a $2 \mathrm{DS}^{4}$.

Respecto al índice cintura-estatura (ICE), se clasificó como obesidad abdominal cuando este se reportó mayor a 0.5 y como normal cuando fue igual o menor a 0.5 .

La medición de la FC se realizó mediante técnica auscultatoria en 3 momentos; la primera, posterior a reposo de 5 minutos, con el niño sentado en posición cómoda; la segunda medición tras ejercicio de intensidad moderada realizado por 5 minutos (subir escaleras a velocidad moderada) y la tercera tras 5 minutos de reposo después del ejercicio. Esta medición fue realizada por enfermeras especialistas en pediatría, quienes colocaron la campana del estetoscopio en el quinto espacio intercostal del lado izquierdo del esternón (línea media clavicular) ${ }^{15}$ durante un minuto. Previo a este procedimiento, la técnica fue estandarizada para evitar al máximo las variaciones intra e intermedidores.

Se capturó la información en una base de datos Excel y el procesamiento de datos se elaboró en el programa estadístico SPSS versión 18. Se realizó estadística descriptiva, se utilizó Kruskall Wallis, con la finalidad de identificar diferencias de medias de FC entre grupos de participantes con peso normal, sobrepeso y obesidad, se utilizó U de Mann Whitney para evaluar diferencias de medias de FC entre pacientes con o sin adiposidad abdominal.

\section{Resultados}

En el estudio se incluyeron 106 niños, la distribución por género fue un 44\% femeninos (47) y un 56\% masculinos (59); la clasificación del estado nutricional, con base en el score Z, una tercera parte (36 niños) se clasificaron con peso normal y el 66\% restante con sobrepeso/obesidad; en relación al ICE, 56 de los participantes (53\%) presentaron obesidad abdominal (Tabla 1).

Tabla 1. Descripción del estado nutricional de la población de acuerdo a ICE y a Score Z de IMC ( $n=106)$

\begin{tabular}{lcccc}
\hline & \multicolumn{2}{c}{ Índice cintura-estatura (ICE) } & Score Z \\
\cline { 2 - 5 } & Normal F (\%) & Obesidad Abdominal F (\%) & Normal F (\%) & Sobrepeso/ Obesidad F (\%) \\
\hline Femenino & $19(40)$ & $28(60)$ & $10(21)$ & $37(79)$ \\
Masculino & $31(53)$ & $28(47)$ & $26(44)$ & $33(56)$ \\
Total & $50(47)$ & $56(53)$ & $36(34)$ & $70(66)$ \\
\hline
\end{tabular}

Frecuencia cardiaca y actividad física en niños con obesidad del Sur de Sonora • J. Valle-Leal et al. 
La edad media de los participantes fue 9 años, el peso promedio de 37 kilogramos, las medias y desviación del resto de variables numéricas se muestran en Tabla 2.

Tabla 2. Descripción de edad y variables somatométricas de escolares que se atienden en un hospital de segundo nivel de atención $(n=106)$

\begin{tabular}{lcccccc}
\hline & Edad & Peso & Talla & Score Z & Per Cin & ICE \\
\hline Media & 8.6 & 37.2 & 134.0 & 2.2 & 70.1 & .519 \\
DE & 1.8 & 12.4 & 11.5 & 2.1 & 13.2 & .082 \\
Mínimo & 6 & 18.6 & 112 & -1.48 & 50 & .38 \\
Máximo & 12 & 70.9 & 158 & 9.38 & 102 & .72 \\
\hline
\end{tabular}

Per Cin: perímetro de cintura, ICE: Índice cintura-estatura, DE: desviación estándar

En las mediciones de la FC se obtuvo una FC basal media de 95 latidos por minuto (lpm), el promedio de la FC post actividad fue de $131 \mathrm{lpm}$ y la FC en reposo post actividad media fue de $107 \mathrm{lpm}$ (Tabla 3).

Tabla 3. Descripción de la frecuencia cardiaca tomada en reposo, post actividad, y post reposo tras actividad en pacientes escolares atendidos en un hospital de segundo nivel $(n=106)$

\begin{tabular}{lccc}
\hline & $\begin{array}{c}\text { FC 1 } \\
\text { en reposo }\end{array}$ & $\begin{array}{c}\text { FC 2 } \\
\text { post actividad física }\end{array}$ & $\begin{array}{c}\text { FC 3 } \\
\text { en reposo tras actividad física }\end{array}$ \\
\hline Media & 94.99 & 131.22 & 106.69 \\
DE & 13.59 & 21.31 & 16.18 \\
Mínimo & 58 & 85 & 64 \\
Máximo & 130 & 196 & 143 \\
\hline
\end{tabular}

Se encontraron diferencias estadísticamente significativas entre medias de FC post actividad y en reposo post actividad, en pacientes con estado nutricional normal, sobrepeso y obesidad de acuerdo a clasificación por score Z de IMC medido por Kruskall Wallis (Tabla 4), también se encontraron diferencias estadísticamente significativas de medias de FC post actividad y post reposo tras actividad entre pacientes con y sin obesidad abdominal, medido por ICE (Tabla 5).

Tabla 4. Diferencia de medias de Frecuencia cardíaca entre pacientes con peso normal, obesidad y sobrepeso de acuerdo al score $Z$ de IMC $(n=106)$

\begin{tabular}{lcccc}
\hline & \multicolumn{3}{c}{ Score Z IMC } & \multirow{2}{*}{} \\
\cline { 2 - 4 } & Normal & Sobrepeso & Obesidad & .721 \\
\hline FC1 Media & 93.5 & 96.08 & 95.68 & $.001^{*}$ \\
FC2 Media & 120.5 & 132.58 & 137.41 & $.000^{*}$ \\
FC3 Media & 99.85 & 103.58 & 111.74 &
\end{tabular}

$\mathrm{p}=$ Kruskall- Wallis, *Significancia estadística

FC1: frecuencia cardiaca en reposo, FC2: frecuencia cardiaca post actividad física, FC3: frecuencia cardiaca en reposo tras actividad física.

Tabla 5. Diferencia de medias de Frecuencia cardiacas en pacientes con y sin obesidad abdominal de acuerdo al ICE $(n=106)$

\begin{tabular}{|c|c|c|c|}
\hline & \multicolumn{2}{|c|}{ ICE } & \multirow[b]{2}{*}{$\mathbf{p}$} \\
\hline & Normal & Obesidad Abdominal & \\
\hline FC1 Media & 94.3 & 95.5 & .644 \\
\hline FC2 Media & 122.9 & 138.6 & $.000^{*}$ \\
\hline FC3 Media & 100.4 & 112.2 & $.000 *$ \\
\hline
\end{tabular}

$\mathrm{p}=$ U de Mann Whitney, *Significancia estadística

ICE: Índice cintura-estatura, FC1: frecuencia cardiaca en reposo, FC2: frecuencia cardiaca post actividad física, FC3: frecuencia cardiaca en reposo tras actividad física. 


\section{Discusión}

En los resultados de este estudio, se encontraron diferencias significativas de medias de FC medidas posterior a actividad física y en reposo tras actividad física, en niños con sobrepeso y obesidad se presentaron frecuencias cardiacas mayores; de igual manera los niños con obesidad abdominal (ICE >0.5) mostraron FC medias mayores tras la actividad física y posterior al reposo tras la actividad. Estos hallazgos se explican por la alteración del Sistema Autonómico que puede presentarse en pacientes con sobrepeso y obesidad, donde predomina la acción simpática sobre la parasimpática ${ }^{15}$, de esta forma tras realizar actividad física los pacientes continúan con FC altas y la recuperación a la basal puede verse limitada ${ }^{16}$.

Estudios anteriores reportan resultados contradictorios sobre la disfunción cardiaca autonómica en niños con obesidad. Agaty et al, reportaron en adultos jóvenes FC mayores posterior a ejercicio físico en sujetos con obesidad en comparación con sujetos con IMC normal ${ }^{17}$. Mansilla-Nilsson et al, no encontraron diferencias en FC en reposo ni tampoco tras ejercicio en niños catalogados con y sin riesgo cardiovascular de acuerdo al índice de cintura- estatura (ICE) ${ }^{18}$. Por otra parte, Tascilar ${ }^{15}$, Vanderlei ${ }^{19}$, Kaufman ${ }^{20}$ y Sekine ${ }^{21}$ informan que en pacientes con obesidad existe una reducción de la actividad parasimpática vagal con un incremento en la actividad simpática.

Los valores de FC media medidos en reposo en pacientes con o sin obesidad no presentaron diferencias significativas. Nuestros resultados concuerdan con los presentados por Paschoal et al, donde se estudiaron a niños brasileños entre 9 y 11 años con y sin obesidad, no se encontró diferencia en FC medias, medidas en reposo ${ }^{10}$. En serie presentada por Rodríguez-Colon et a ${ }^{22}$, realizada en 616 niños de quinto grado de primaria en el estado de Pensilvania, Estados Unidos de Norteamérica, se encontraron diferencias significativas de medias de FC en participantes con IMC normal, con sobrepeso y obesidad. Resultados similares fueron presentados por Sekine et al., tras estudiar a niños entre 8 y 9 años de edad en Japón, en este grupo los pacientes con obesidad presentaron medias de FC en reposo significativamente mayores que en niños con IMC normal ${ }^{21}$. Estas discrepancias pueden deberse a la diferencia del tamaño de la muestra, a la conformación de los grupos de acuerdo al estado nutricional, o también al tiempo en que el grupo de pacientes estudiados ha estado expuesto a la obesidad.

Faltan estudios, sobre todo con diseños longitudinales donde se dé un seguimiento a pacientes con alteraciones en la FC en reposo y tras el ejercicio, además de la asociación a marcadores bioquímicos que puedan ser sensibles para la detección temprana de alteraciones cardiovasculares como; enfermedad coronaria, hipertensión, síndrome metabólico, entre otras.

El ejercicio físico es uno de los principales recursos terapéuticos utilizados en la obesidad. Existen publicaciones que reportan que el ejercicio puede mejorar la modulación autonómica cardiaca en niños con obesidad ${ }^{23-25}$, por lo que es importante que se evalúe integralmente al niño, con la finalidad de establecer un plan de actividad física, acorde a las capacidades funcionales de cada niño.

El marcado aumento de la FC después de la actividad física en los niños con sobrepeso y obesidad, debe ser tomado en cuenta como signo de alarma temprano de disfunción cardiovascular, estos pacientes deben tener seguimiento para referencias futuras, ya que pueden presentar un inicio precoz de alguna alteración cardiaca relacionada con el sobrepeso/obesidad y/o la obesidad abdominal.

El método de medición de la FC en este estudio se realizó con técnica básica de auscultación en consultorio de pediatría, mediciones que se pueden realizar en cualquier centro de atención médica; es significativo considerar las mediciones en reposo de la FC, PA en todo niño que acude a consulta, la comparación de estas con el patrón de referencia para su edad de acuerdo al género, la detección de alteraciones tempranas, nos otorgan la oportunidad de intervención y prevenir el desarrollo de enfermedades cardiovasculares, sobre todo en pacientes con riesgo aumentado para desarrollarlas como son aquellos con sobrepeso y obesidad. El personal médico y de enfermería debe establecer medidas preventivas de salud en este grupo poblacional, para desarrollarse principalmente en escenarios donde estos permanecen cautivos, como escuelas o guarderías. La evaluación del estado nutricional y de la adecuada función del sistema cardiovascular es importante, para el establecimiento de riesgos a la salud desde 
etapas tempranas de la vida, y de esta manera poder iniciar medidas preventivas efectivas que disminuyan las tasas de obesidad, enfermedades metabólicas y padecimientos cardiovasculares.

Algunos de los factores pendientes por analizar en este estudio fueron los hábitos alimenticios, la actividad física promedio del menor y el tiempo aproximado que el niño ha presentado sobrepeso u obesidad; ya que estos son factores que podrían modificar la respuesta cardiaca a la actividad física. Existen métodos con mayor precisión que el auscultatorio para la medición de la frecuencia cardiaca, como puede ser el electrocardiograma, dispositivos avanzados ${ }^{26}$, sin embargo, no contamos con financiamiento para la adquisición de dichos instrumentos.

Estos resultados pueden ser útiles para el personal de enfermería, médicos, nutriólogos, especialistas en ejercicio, al momento de evaluar estado nutricional, salud cardiovascular y estructurar rutinas de actividad física para personas con sobrepeso u obesidad, al tomar en consideración las alteraciones de la frecuencia cardiaca presentes en este grupo de pacientes.

\section{Responsabilidades Éticas}

Protección de personas y animales. Los autores declaran que en esta investigación no se han realizado experimentos en seres humanos ni en animales.

Confidencialidad. Los autores declaran que este trabajo no contiene ningún dato confidencial de los participantes.

Conflicto de intereses. Los autores declaran no tener conflicto de intereses.

Financiamiento. Ninguno

Agradecimientos. Al personal directivo, coordinadoras del curso postécnico de enfermería pediátrica, personal médico y de enfermería adscritos al servicio de consulta de pediatría del Hospital General regional Número Uno del IMSS en Sonora.

\section{Referencias}

1. Kaplan NM, Chatterjee K, Fuster V, et al. Errores frecuentes en cardiología. Barcelona: Ediciones Médicas; 2009. p. 14-15

2. Fernández-Jaimes ME, Zárate-Grajales RA, Ochoa-Cervantes JM, et al. La evaluación de la calidad de los signos vitales como indicador de proceso en la Gestión del Cuidado de Enfermería. Rev. mex. enferm. cardiol. 2010; 18 (3): 65-70.

3. CENETEC. Prevención y diagnóstico de sobrepeso y obesidad en niños y adolescentes en el primer nivel de atención. México: Secretaría de Salud; 2008 [Consultado 03 marzo 2016]. Disponible en: https://bit.ly/2Ptw5Da

4. Torres-Tamayo M, Aguilar-Herrera BA, Altamirano-Bustamante N, et al. Consenso de expertos sobre prevención, diagnóstico y tratamiento de la obesidad en edad pediátrica. Bol Med Hosp Infant Mex. 2015; 72 (Suple 1): 1-28 [Consultado 16 noviembre 2016]. Disponible en: https://bit.ly/2zSn8IE

5. Gutiérrez JP, Rivera-Dommarco J, Shamah-Levy T, et al. Encuesta Nacional de Salud y Nutrición 2012. Resultados Nacionales. Cuernavaca, México: Instituto Nacional de Salud Pública; 2012.

6. Hernández-Ávila M, Rivera-Dommarco J, Shamah-Levy T, et al. Encuesta Nacional de Salud y Nutrición de Medio Camino 2016, Informe Final de Resultados. Cuernavaca, México. Instituto Nacional de Salud Pública; 2016.

7. Paschoal MA, Fernades-Trevizan P, Folco-Scodeler N. Variabilidad de la frecuencia cardiaca, lípidos y capacidad física de niños obesos y no obesos. Arq Bras Cardiol. 2009; 93 (3): 235-241. http://dx.doi.org/10.1590/S0066-782X2009000900007

8. Claudino-Rossi R, Marquez-Vanderlei LC, Campagnolo-Real AC, et al. Impact of obesity on autonomic modulation, heart rate and blood pressure in obese young people. Auton Neurosci. 2015; 193: 138-41. https://doi.org/10.1016/j.autneu.2015.07.424

9. Rodas G, Pedret-Carballido C, Ramos J, et al. Variabilidad de la frecuencia cardiaca: concepto, 
medidas y relación con aspectos clínicos (I). Arch. med. deporte. 2008; 25 (123): 41 -7 [Consultado 21 septiembre 2016]. Disponible en: https://bit.ly/2DFK6XU

10. Paschoal MA, Fontana CC. Método del umbral de variabilidad de la frecuencia cardiaca aplicado en preadolescentes obesos y no obesos. Arq Bras Cardiol. 2011; 96 (6): 450-56.

http://dx.doi.org/10.1590/S0066-782X2011005000052

11. Liao D, Rodríguez-Colón SM, He F, et al. Childhood Obesity and Autonomic dysfunction: Risk for Cardiac Morbidity and Mortality. Curr Treat Options Cardiovasc Med. 2014; 16 (10); Article 342. pp.1-13. https://doi.org/10.1007/s11936-014-0342-1

12. Cote AT, Harris KC, Panagiotopoulos C, et al. Childhood Obesity and Cardiovascular Dysfunction. J Am Coll Cardiol. 2013; 62 (15): 1309-19. http://dx.doi.org/10.1016/j.jacc.2013.07.042

13. Aparicio MR, Estrada LA, Fernández C, et al. Manual de antropometría. $2^{\text {da }}$ ed. Distrito Federal, México: Instituto Nacional de Ciencias Médicas y Nutrición Salvador Zubirán. 2004.

14. Cobo D, Daza P. Signos vitales en pediatría. Rev Gastrohnup. 2011; 13 (Supl1): 58-70 [Consultado 04 abril 2017]. Disponible en: https://bit.ly/2nM7311

15. Taş̧̧ılar M, Yokuşoğlu M, Boyraz M, et al. Cardiac Autonomic Fuctions in Obese Children. J Clin Res Pediatr Endocrinol. 2011; 3 (2): 60-4. https://dx.doi.org/10.4274\%2Fjcrpe.v3i2.131

16. Suazo-Fernández R, Fernández-Dávila FV. Actividad física, condición física y factores de riesgo cardio-metabólicos en adultos jóvenes de 18 a 29 años. An. Fac. med. 2017; 78 (2): 145-9.

17. El Agaty SM, Kirmani A, Labban E. Heart rate variability analysis during inmediate recovery from exercise in overweight/obese healthy young adult females. Ann Noninvasive Electrocardiol. 2017; 22 (3): e12427. https://doi.org/10.1111/anec.12427

18. Mansilla-Nilsson M, Muñoz-Sepúlveda F, Enríquez-Schmidt M, et al. Frecuencia cardiaca postejercicio aeróbico y anaeróbico en niños con y sin riesgo cardiovascular. Rehabilitación (Madr. Internet). 2014; 48 (3): 144-50. https://doi.org/10.1016/j.rh.2013.12.005

19. Marques-Vanderlei LC, Pastre CM, Freitas-Junior IF, et al. Analysis of cardiac autonomic modulation in obese and eutrophic children. Clinics (Sao Paulo). 2010; 65 (8): 789-92. https://dx.doi.org/10.1590\%2FS1807-5932201000080009

20. Kaufman CL, Kaiser DR, Steinberger J, et al. Relationships between heart rate, variability, vascular function, and adiposity in children. Clin Auton Res. 2007; 17 (3): 165-71. https://doi.org/10.1007/s10286-007-0411-6

21. Sekine M, Izumi I, Yamagami T, et al . Obesity and Cardiac Autonomic Nerve Activity in Healthy Children: Results of the Toyoma Birth Cohort Study. Environ Health Prev Med. 2001; 6 (3): 149-53. Disponible en: https://dx.doi.org/10.1007\%2FBF02897962

22. Rodríguez-Colón SM, Bixler EO, Li X, et al. Obesity is associated with impaired cardiac autonomic modulation in children. Int J Pediatr Obes. 2011; 6 (2): 128-34. https://dx.doi.org/10.3109\%2F17477166.2010.490265

23. Gutin B, Barbeau P, Litaker MS, et al. Heart Rate Variability in Obese Children Relations to Total Body and Visceral Adiposity, and Changes with Physical Training and Detraining. Obes Res. 2000; 8 (1): 12-9. https://doi.org/10.1038/oby.2000.3

24. Nagai N, Moritani T. Effect of physical activity autonomic nervous system function in the lean and obese children. Int J Obes Relat Metab Disord. 2004; 28 (1): 27-33. https://doi.org/10.1038/sj.ijo.0802470

25. Gamelan FX, Baquet G, Berthoin S, et al. Effect of high intensity intermittent training on heart rate variability in prepubescent children. Eur J Appl Physiol. 2009; 105 (5): 731-8. https://doi.org/10.1007/s00421-008-0955-8

26. Vascocellos FVA, Seabra A, Cunha F, et al. Heart rate variability assesment with fingertip photoplethysmography and polar RS800cx as compared with electrocardiography in obese adolescents. Blood Press Monit. 2015; 20 (6): 351-60. https://doi.org/10.1097/MBP.0000000000000143 